\title{
A REVIEW OF CURRENT STATUS OF TAMIL NADU UNDER REVISED NATIONAL TUBERCULOSIS CONTROL PROGRAM
}

Dr.V.Uma Devi, Professor\& Head,

Department of General Medicine, Shri Sathya Sai Medical College \& Research Institute, Sri Balaji Vidyapeeth, Nellikuppam, Kancheepuram Dist., Tamil Nadu - 603 108, India.

\section{Introduction}

Tuberculosis remains worldwide public health problem despite the fact the causative organism was discovered more than 100 years ago and highly effective drugs are available. Tuberculosis is a top infectious disease killer in the world. It occurs in every part of the world. The largest number of TB cases occurred in South East Asian and Western Pacific Region. About $80 \%$ of total reported cases occurred in 22 countries in 2014 with Africa topping the list with a prevalence of 281/ 1 lakh. (Compared to world average 133/1lakh).

Tuberculosis is one of the leading causes of mortality in India- killing - 2 persons every three minute, nearly 1,000 every day. Currently India has a TB prevalence of 197/1 lakh. Around 2200 new cases of tuberculosis are reported in India in 2014(ranging from $2000-2300$ in various regions of India). Around 220 people have died in 2014 in India due to Tuberculosis alone. TB affects all ages and both sexes. It is a social disease with medical aspects and is a measure of social welfare. ${ }^{1}$

\section{Nationwide Control Measures}

TB control was started in way back in 1962 with National Tuberculosis Control Programme. Domiciliary treatment was recommended under NTCP with a standard drug treatment for 12-18 months. Priority was given to newly detected patients than re-treatment cases and drugs supplied free of cost. Chemotherapy of TB underwent revolutionary changes in the seventies owing to the availability of two well-tolerated and highly effective drugs - Rifampicin and Pyrazinamide. These drugs allowed short course chemotherapy (SCC) and made it possible to simplify treatment and reduce its duration. By 1992 under revised national tuberculosis control programme the short course chemotherapy under direct observation was made the corner stone of the control programme. The strategy of Directly Observed Treatment, Short-course (DOTS) is based largely on research done in India in the field of TB over the past 35 years and is adopted in $>180$ countries.

\section{Revised National Tuberculosis Control Program (RNTCP)}

In Tamil Nadu, Revised National Tuberculosis Control Program(RNTCP) is implemented since 2001.Today RNTCP covers 33 districts in Tamil Nadu with a total population of 754.72 lakhs. In the year 2013 about 6, 59,389 presumptive TB cases were screened and 80,407 patients were registered for TB. There are $220 \mathrm{~TB}$ units and 784 Designated Microscopy Centres across the state to carry on the diagnosis and treatment of TB. The total TB case notification in Tamil Nadu was 109 cases per lakh per year for the year 2013 . 


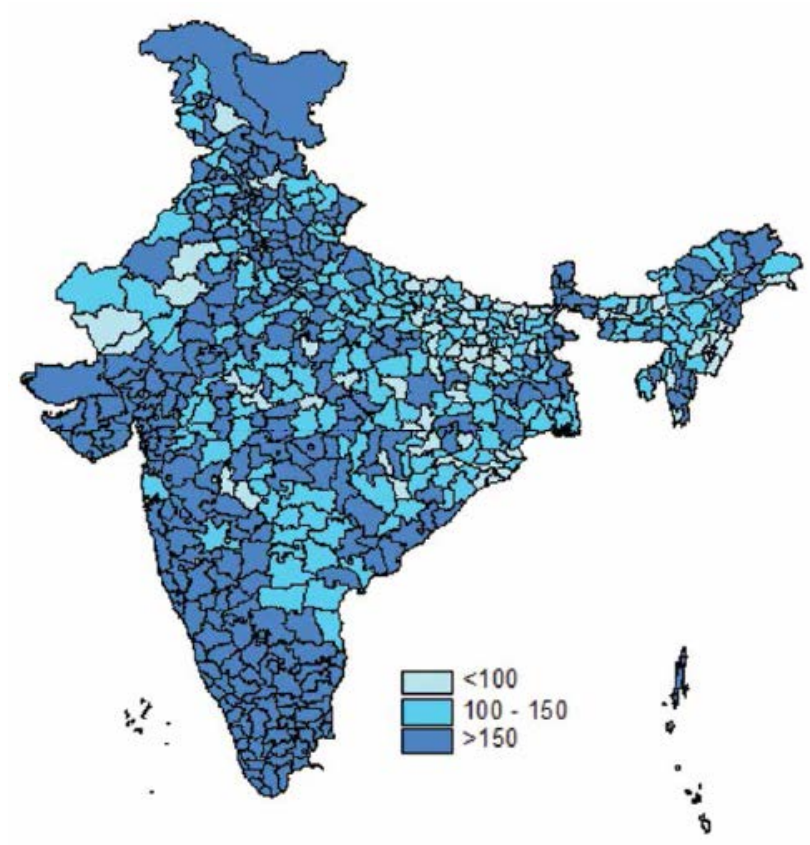

Fig 1: TB suspects examined/1lakh population

One of the major policy decision taken by RNTCP in the year 2010 is to change the focus of the NSP case detection objective of at least $70 \%$ to the concept of universal access to good quality care for TB patients. There is now global consensus that the twin objectives of $70 / 85$ alone is not enough to achieve adequate reduction of TB transmission and reduction in disease burden at the pace with which epidemiological impact is expected (Fig 1).

With the aim of improving the collection of patient care information, in May 2012 India declared TB to be a notifiable disease (Fig 2). This means that in future all private doctors, caregivers and clinics treating a TB patient must report every case of TB to the government. for this purpose the government uses a portal NIKSHAY. By 2014, $1845 \mathrm{~TB}$ patients are notified by the private sector.

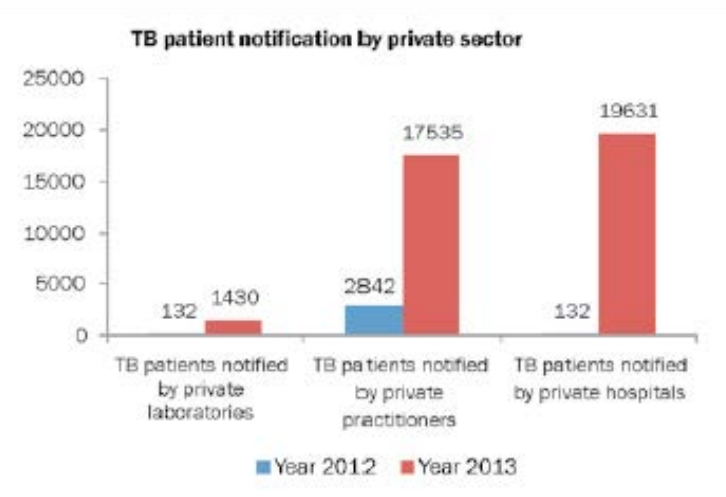

Fig 2: Incidence of TB cases notified /1lakh population in India
In 2014, TamilNadu has an incidence of 108 TB cases / 1 lakh population. The Pediatric cases out of total new cases are $3385(5 \%)$. the registration and the start of treatment are satisfactory as $83 \%$ of smear positive cases are started on treatment within 7 days of diagnosis. As the Intensive Phase is given in most parts in PHCs, initial follow up and end of treatment follow up are good. $82 \%$ of cases are tested for follow up sputum at the end of the treatment within 7 days of last dose.

The current upsurge of tuberculosis in previously controlled western countries is due to HIV infection. TB is the most common opportunistic infection in HIV and both disease virtually co-exist that in the RNTCP all patients receiving ATT are counseled for HIV testing and treated according to HIV status. In 2014, 110 cases of TB with HIV positive are reported in India (8.3/1lakh) ranging from 96 to120 in different regions. in Tamilnadu, 92\% of the TB patients know the HIV status and $6 \%$ are HIV positive. Number of HIV infected TB patients who are put on ART are $79 \%$ and those put on cotrimoxazole prevention treatment is $90 \% .^{2}$

Among the smear positive cases, $84 \%$ cure rate is achieved in Tamil Nadu. $5 \%$ of the smear positive patients died. $2 \%$ failure and $4 \%$ defaulters were reported. $64 \%$ cure rate is achieved in retreatment cases with $2 \%$ going for DOTS plus treatment

The pitfalls in RNTCP are that ${ }^{(1)}$ The Programme mostly emphasis on identifying the case and registering for the treatment and does not emphasize on health education for continued treatment and follow up.

(2) Continuous and adequate supply of all drugs is of paramount importance in the success of DOTS. as complete treatment with DOTS reduces the emergence of resistance thereby promoting the individual's as well as the country's economy as the treatment with DOTS plus is highly costly and tiresome .

${ }^{(3)}$ In case of category I failure instead of adding one other drug it would be better to treat as per drug sensitivity. Also the current $2^{\text {nd }}$ line drugs used in regimen are available in the market and most commonly used for various other illnesses which may be a cause for resistance.

(4) Training of doctors in diagnostic skills is necessary to correctly exclude other mimicking conditions like silicosis and finding out the probable cases as a considerable prevalence and spread are attributable to non-diagnosis. 
The uncertainty of sputum smear negative cases may continue to spread infection in the community, if their $\mathrm{x}$ -rays are found inconclusive. ${ }^{(5)}$ Enabling public-private partnership to prevent dropouts and emergence of resistance the social stigma associated with TB should be curbed with appropriate IEC and political commitment for TB has to be increased. Community participation and the role of NGOs should be increased further for the sustained implementation and success of DOTS.

\section{REFERENCES}

1. Annual report of RNTCP-2014.

2. Global burden of Tuberculosis-2015 by WHO . 